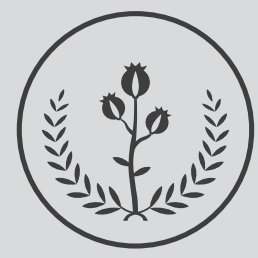

FUCS

\section{Re

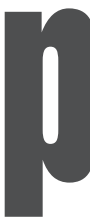

\title{
Banco de leche humana (BLH): una estrategia de apoyo para la atención de neonatos
}

\section{Human milk bank (HMB): a support strategy for newborn care}

${ }^{a}$ Médico Veterinario Zootecnista, Epidemiologia Msc., PhD(C) Gestión de Proyectos. Director Proyectos e Investigaciones Fundación Nacional Somos - FNS, Fundación Universitaria de Ciencias de la Salud. Bogotá DC, Colombia.

${ }^{b}$ Administración en Salud con énfasis en Seguridad Social, Epidemiología y Auditoria de Servicios de Salud. Maestría en Salud Pública y Desarrollo Social. Directora Nacional de Programas de Salud de la EPS Medimás. Bogotá DC, Colombia.

'Optometría, Docencia Universitaria, Especialista en Gerencia de Instituciones en Salud, Auditoria en Salud. Maestría en Salud Pública y Desarrollo Social, Gerente de IPS privada en Fusagasugá Cundinamarca Colombia.

\section{R ES U M EN}

Introducción: la lactancia materna ha demostrado grandes beneficios para el recién nacido. En vista de que hay factores que afectan la correcta lactancia, en 2007 se crearon los bancos de leche materna y Colombia hace parte de esta iniciativa en pro de la niñez. Objetivo: identificar la utilización del banco de leche humana (BLH) de la ESE Hospital San Rafael de Fusagasugá, Colombia entre julio 2012 y junio 2016. Metodología: estudio descriptivo de corte transversal, con muestra de 1282 nacidos en el período que utilizaron el BLH. Resultados y discusión: entre los factores que llevaron al uso del BLH en estos menores están la baja producción de leche en la madre en 53,7\%, cirugía materna 3,5\%, mamá con bajo nivel cognitivo 0,2\%, succión leve o pobre en $9,6 \%$, madre ausente $0,4 \%$, recién nacido que requirió hospitalización 4,6\%, pezón invertido o plano $2,5 \%$ y labio leporino 0,5\%. Conclusión: se pudo determinar que 22,7\% de los niños que nacieron en el municipio necesitaron

\section{INFORMACIÓN DEL ARTÍCULO}

Historia del artículo:

Fecha recibido: enero 22 de 2020

Fecha aceptado: julio 28 de 2020
Autor para correspondencia.

Sr. Héctor Fabio Restrepo

hfrestrepo@fucsalud.edu.co
DOI

10.31260/RepertMedCir.01217372.1006 
el servicio del banco de leche materna, logrando visualizar las bondades del mismo, brindando la oportunidad para que lo pudieran utilizar en la misma zona de nacimiento sin necesidad de desplazarse, evitando las subsiguientes consecuencias que ello desencadena en la familia y el recién nacido.

Palabras clave: lactancia materna, bajo peso, banco de leche.

(C) 2021 Fundación Universitaria de Ciencias de la Salud - FUCS. Este es un artículo Open Access bajo la licencia CC BY-NC-ND (http://creativecommons.org/licenses/by-nc-nd/4.0/).

\section{A BSTRACT}

Introduction: breastfeeding has shown great benefits for the newborn. In view of the fact that there are factors affecting proper breastfeeding, nursing mothers' milk banks were created in 2007 and Colombia is part of this initiative on behalf of children. Objective: to identify the use of the human milk bank (HMB) at ESE Hospital San Rafael in Fusagasugá, Colombia between July 2012 and June 2017. Methodology: descriptive cross-sectional study, with a sample of 1282 newborns delivered in said period using HMB. Results: among the factors that led to the use of HMB in these children are the mother's low milk production in $53.7 \%$, maternal surgery $3.5 \%$, mother with low cognitive level $0.2 \%$, mild or poor suction $9.6 \%$, absent mother $0.4 \%$, newborn requiring hospitalization $4.6 \%$, inverted or flat nipple $2.5 \%$ and cleft lip $0.5 \%$. Conclusion: it could be determined that $22.7 \%$ of the children born in the municipality needed the service of the mother's milk bank, managing to visualize the benefits of it, providing the opportunity for them to use it in the same area of birth without having to move, avoiding the subsequent consequences that this triggers in the family and newborn.

Key words: breastfeeding, low weight, milk bank.

(C) 2021 Fundación Universitaria de Ciencias de la Salud - FUCS. This is an open access article under the CC BY-NC-ND license (http://creativecommons.org/licenses/by-nc-nd/4.0/).

\section{INTRODUCCIÓN}

La lactancia materna ha tomado especial importancia en los últimos años, gracias a los beneficios que aporta a madres e hijos en campos que van desde el aspecto médico hasta los psicoactivos y económicos. La Organización Panamericana de la Salud (OPS) anota: "unas prácticas de alimentación adecuadas son fundamentales para la supervivencia, el crecimiento, el desarrollo, la salud y la nutrición de los lactantes y niños en cualquier lugar del mundo". ${ }^{1}$ A pesar de ello, de acuerdo con el reporte de Sáenz y col. sólo “35\% de lactantes de todo el mundo son alimentados exclusivamente con leche materna durante los primeros cuatro meses de vida". ${ }^{2}$ Existe evidencia que demuestra que la alimentación con leche materna posee ventajas sustanciales para los neonatos, tanto prematuros como de bajo peso al nacer, en comparación con la leche de formula. ${ }^{3-7}$ La leche materna según afirman Rodríguez y col. "tiene una naturaleza dinámica ajustada a las necesidades del niño en crecimiento y que no solo aporta componentes nutritivos sino también factores bioactivos necesarios para el desarrollo infantil". ${ }^{8}$ Es importante considerar también que la "lactancia materna, la nutrición y el desarrollo en las etapas tempranas de la vida son claves para alcanzar prácticamente todos los objetivos de desarrollo del milenio" de acuerdo con lo referido por la OMS/OPS ${ }^{9}$; de igual manera, la OMS define que la lactancia natural "es una forma sin parangón de proporcionar un alimento ideal para el crecimiento y el desarrollo de los lactantes". ${ }^{2}$

La definición clásica de la lactancia exclusiva la consideran los autores como "un infante que consume leche materna sin suplementación de ningún tipo, excepto por vitaminas, minerales y medicamentos"10; por otra parte, la lactancia natural además de ser la fuente más pura de alimentación logra disminuir el costo de los cuidados y mejora las condiciones de salud de los niños que la consumen. ${ }^{2}$ A nivel de la maduración gastrointestinal se reporta en la literatura que está favorecida por la leche materna en comparación con la de formula. ${ }^{11,12}$ En 1997 la Academia Americana de Pediatría (AAP) publicó la declaración de la política sobre lactancia materna y el uso de leche humana. ${ }^{10}$ Sin embargo, se considera que existe un número pequeño de condiciones de salud del recién nacido o de la madre que conllevan al no amamantamiento de manera temporal o permanente. $^{4,12}$ Además, como la afirman Rodríguez y col. "la gran difusión de fórmulas lácteas que acompañaron el crecimiento de la industria, la gran disponibilidad de biberones y la introducción de otros alimentos han traído una disminución de la lactancia materna en la actualidad". ${ }^{8}$ Los BLH han surgido como una medida para disminuir los índices de morbimortalidad infantil "mediante la promoción, protección y apoyo a la lactancia materna y el 
procesamiento, control de calidad y suministro de la leche humana a los neonatos hospitalizados, que garantizan la seguridad alimentaria y nutricional."13 Los BLH son entonces una estrategia de intervención y apoyo para la supervivencia de neonatos impulsada por los estados y los gobiernos para disminuir los índices de mortalidad infantil, promocionando la lactancia materna en sus territorios como un apoyo nutricional ${ }^{14,15}$ y como estrategia para administrar leche cuando la madre no la puede proveer. $^{5}$

La implementación de un banco de leche tiene la finalidad de asegurar la lactancia materna exclusiva ${ }^{16}$, articulándose con los objetivos de desarrollo sostenibles en sus componentes nutricionales y de protección, cuyo impacto a nivel mundial obliga a su cumplimiento. Teniendo en cuenta estos beneficios, los estados y gobiernos han desplegado programas y estrategias para fomentar la lactancia materna e incluso acuerdos de cooperación internacional como la Red Iberoamericana de Bancos de Leche Humana, en "el contexto de la iniciativa regional para la erradicación de la desnutrición infantil en América Latina y el Caribe" ${ }^{\prime 17}$, lo cual tiene como principal objetivo: “apoyar la implantación de por lo menos un banco de leche en cada país iberoamericano." ${ }^{\prime 18}$

La Red Iberoamericana de Bancos de Leche Humana (BLH) nace en 2007 como estrategias para lograr los objetivos de desarrollo del milenio y reducir la mortalidad infantil. Los primeros integrantes de la red fueron Argentina, Brasil, España, Bolivia, Uruguay, Paraguay y Venezuela, en el marco de la XVII Cumbre Iberoamericana que se celebró en Santiago de Chile ese año., ${ }^{917}$ De acuerdo con la Unicef, los centros lactarios son un componente importante donde las madres deben contar con un ambiente tranquilo en el cual podrán almacenar su leche y conservarla de manera adecuada. ${ }^{14,19}$ En 2005 Colombia con la firma de la carta de Brasilia se vinculó a la red implementando esta estrategia, con el ánimo de garantizar la seguridad alimentaria y nutricional de los prematuros internados en las unidades de cuidados intensivos. ${ }^{20,21}$ Hace parte de la política de primera infancia que Colombia en cooperación con el Ministerio de Salud de Brasil desde 2011 viene avanzando en la implementación de BLH que se inició en los hospitales Federico Lleras Acosta de Ibagué y la ESE Hospital San Rafael de Fusagasugá. A los 10 meses se lograron 297 mujeres donantes con un total de 530 litros de leche humana, beneficiando a 513 niños con bajo peso neonatal, lo que demuestra el gran beneficio de esta implementación en el país. ${ }^{22}$

Enmarcado en el plan decenal de lactancia materna 20102020 y la estrategia de atención integral a la primera infancia "de cero a siempre", los bancos de leche en Colombia han sido un medio para disminuir las cifras de morbimortalidad infantil, mejorar la salud materna e ir un paso adelante en cuanto a programas que incentiven la práctica de la lactancia infantil. De la misma manera, los BLH garantizan a los neonatos que demandan complementación alimentaria el acceso de primera mano a la leche humana con calidad certificada, amortizando los costos que se generan por compra y suministro de fórmulas lácteas $\mathrm{y}$ productos industrializados. ${ }^{20}$

Los principales beneficiarios de los BLH son neonatos (28 días o menos desde su nacimiento) hospitalizados en unidades de cuidado intermedio o intensivo neonatal, norma que se aplica en doce de los trece hospitales que cuentan con BLH en Colombia (Bogotá, Cali, Cartagena, Cúcuta, Facatativá, Fusagasugá, Ibagué, Medellín, Pasto, Popayán, Santa Martha, Valledupar y Zipaquirá), siendo la ESE Hospital San Rafael de Fusagasugá la única que carece de unidad de cuidado intensivo neonatal. Es en desarrollo de este acuerdo y enmarcado en la política y el plan nacional de seguridad alimentaria nutricional, el plan decenal para la promoción, protección y apoyo a la lactancia materna y la estrategia de cero a siempre ${ }^{20}$, que en Colombia se ha implementado una red de bancos de leche humana desde 2011. Esta política ha sido adoptada por el actual gobierno en el Plan Nacional de Desarrollo 2014-2018, todos por un nuevo país, que busca mejorar las condiciones nutricionales de la población colombiana ${ }^{23}$, por medio de diferentes acciones entre las que se cuenta la consolidación de la estrategia de BLH en Colombia.

Se entiende que los beneficiarios de los BLH en Colombia son "prioritariamente niños prematuros y los que nacen con bajo peso (menos de 2.500 gramos), sepsis neonatal, deficiencias inmunológicas, diarreas recurrentes, los sometidos a cirugía, portadores de alergias a proteínas heterólogas y bebés hospitalizados menores de 6 meses con lactancia materna exclusiva." ${ }^{20}$ Dadas las condiciones de priorización, la leche que se procesa en los BLH está destinada para beneficiar a los neonatos hospitalizados en unidades de cuidado intermedio e intensivo, siendo necesario que los BLH se ubiquen en "hospitales o clínicas de segundo, tercero o cuarto nivel de atención, que presten atención materno e infantil con unidad de neonatos, certificados o en proceso de certificación por parte de la Institución Amiga de la Mujer y la Infancia Integral - IAMII". ${ }^{20}$

Formular la pregunta sobre el uso del Banco de Leche de Fusagasugá resulta pertinente, pues como se ha venido exponiendo los BLH están concebidos para la atención de niños y niñas que presenten ciertas características como bajo peso al nacer, que sean prematuros o estén hospitalizados, siendo necesario entonces que esté situado en un hospital que cuente con el servicio de unidad de atención de cuidado intermedio o intensivo neonatal. Esta condición no aplica para el BLH de la ESE Hospital San Rafael de Fusagasugá, pues al no contar con este tipo de unidad especializada, esta investigación busca identificar los usos del BLH partiendo de esta especificidad, es decir, se busca hacer una caracterización de los beneficiarios del BLH desde la inexistencia de unidad neonatal. El objetivo primordial de la presente investigación es por tanto el identificar los usos del Banco de Leche Humana de la ESE Hospital San Rafael de Fusagasugá en el periodo comprendido entre julio 2012 a junio de 2017. 


\section{MATERIALES Y MÉTODOS}

Se planteó un estudio de tipo cuantitativo descriptivo, tomando todos los neonatos que han sido beneficiarios del banco de leche del municipio de Fusagasugá desde el año 2012 hasta julio de 2016, definiendo las características demográficas y los factores que llevaron a su utilización. Los criterios de inclusión fueron la edad del beneficiario (neonatos de 1 a 28 días de nacido), los hospitalizados en la ESE Hospital San Rafael de Fusagasugá, tener consentimiento informado de uso del BLH y que la medición del beneficio esté dentro del periodo seleccionado.

El análisis estadístico se llevó a cabo en el programa estadístico SPSS para Windows, después de la tabulación en el programa excel, donde se definieron espacios para cada una de las variables establecidas; este análisis consistió en la medida de frecuencias de tendencia central y de dispersión para el caso de las variables cuantitativas. Para las cualitativas se procedió a medidas de frecuencia y porcentaje con sus correspondientes intervalos de confianza, se llevó a cabo correlación simple entre variables demográficas y factores de utilización del BLH. Teniendo en cuenta las características del estudio sin llevar a cabo ninguna intervención y siguiendo los lineamientos de la resolución 8430, se consideró sin riesgo. Para garantizar el anonimato de los mismos no se tuvieron en cuenta datos sensibles de localización como nombre, dirección o teléfono, ni se llevó a cabo seguimiento.

\section{RESULTADOS GENERALES}

En el periodo comprendido entre junio 2012 y junio 2016 nacieron un total de 5647 niños en el municipio de Fusagasugá de los cuales 99,8\% ocurrieron en la cabecera municipal; de estos 48,5\% fueron de género femenino; en cuanto al tipo de parto $31,5 \%$ se llevó a cabo por cesárea y $68,2 \%$ de forma espontánea, solo $0,4 \%$ fue instrumentado. Se presentaron 30 partos dobles, 2 triples y 1 cuádruple, el resto fue simple. La edad de la madre al momento del parto fue 19 años en 7,9\%, seguida de 20 años con 7,2\% y 22 años en $6,7 \%$. En cuanto a nivel educativo que primó, está la media académica o básica con $46 \%$ seguida de básica secundaria en $25,1 \%$. El sitio de residencia fue la cabecera municipal en $67,2 \%$, mientras que en la zona rural se ubicó $24,5 \%$ y el restante $8,3 \%$ se localizó en pequeños centros poblados; el régimen subsidiado fue el más frecuente con $62,1 \%$, mientras el contributivo fue $34,7 \%$.

\section{Nacidos que utilizaron el servicio de banco de leche materna}

De todos los nacidos vivos atendidos en el periodo del estudio, 1282 fueron los usuarios directos del banco de leche materna que corresponde a 22,7\%. La distribución de usuarios del BLH por año fue: en 2012, 16,8\%, 2013 fue $27,5 \%, 2014,27 \%$; 2015, 20,7\% y 2016, 7,9\%; de estos $55,4 \%$ corresponden a género masculino, la media de edad gestacional fue 38,4 semanas con una mínima de 24 y máxima de 42, la media de peso estuvo en 3125 gramos y la talla media fue $50 \mathrm{~cm} ; 133(10,4 \%)$ presentaron bajo peso al nacer, 5,2\% fueron prematuros y $0,5 \%$ nacieron con algún tipo de enfermedad infecciosa.

Dentro de los factores que llevaron al uso del BLH en estos menores se encuentran la baja producción de leche materna en $53,7 \%$, cirugía materna 3,5\%, mamá con bajo nivel cognitivo $0,2 \%$, succión leve o pobre 9,6\%, madre ausente $0,4 \%$, recién nacido que requirió hospitalización 4,6\%; presencia de pezón invertido o plano 2,5\% y labio leporino $0,5 \%$. La estancia hospitalaria varió entre 1 y 21 días siendo la media de 1,5 días. El suministro de leche materna estuvo en el rango medio de 202 cc, con una prescripción media de 30 cc diarios (tabla 1).

Teniendo en cuenta el género se encontró que el bajo peso al nacer ocurrió más en el femenino con 51,9\%. La presencia de enfermedad infecciosa se observó en el género masculino en $83,3 \%$. Hubo más casos de gemelares en los hombres que en las mujeres $(55,4 \%$ vs $43,1 \%)$. La baja producción láctea se vió más en las madres de hijos masculinos (56,5\%); los de género masculino presentaron mayor necesidad de ser hospitalizados $(64,4 \%)$. De los casos con pezón invertido o plano fue más frecuente en madres de recién nacidos de género masculino $(56,3 \%)$ y el labio leporino se presentó en ambos géneros en porcentaje similar $50 \%$.

Tabla 1. Condiciones de los niños que recibieron leche del BLH

\begin{tabular}{|lcc|rc|}
\hline & \multicolumn{2}{c|}{ Sí } & \multicolumn{2}{c|}{ No } \\
\hline Enfermencia & $\%$ & Frecuencia & $\%$ \\
Gemelares & 6 & 0,5 & 1276 & 99,5 \\
Baja producción & 17 & 1,3 & 1265 & 98,7 \\
Cirugía materna & 688 & 53,7 & 593 & 46,3 \\
Bajo nivel cognitivo & 45 & 3,5 & 1237 & 96,5 \\
Succión leve o pobre & 123 & 9,6 & 1159 & 90,4 \\
Madre ausente & 5 & 0,4 & 1277 & 99,6 \\
RN hospitalizado & 59 & 4,6 & 1223 & 95,4 \\
Pezón invertido o plano & 32 & 2,5 & 1250 & 97,5 \\
Parto extrahospitalario & 7 & 0,5 & 1275 & 99,5 \\
Labio leporino & 6 & 0,5 & 1276 & 99,5 \\
Bajo peso al nacer & 133 & 10,4 & 1149 & 89,6 \\
\hline
\end{tabular}

Fuente: los autores. 


\section{CONCLUSIONES}

El presente estudio pretendió determinar la frecuencia de uso y los factores que influyeron para recurrir al banco de leche materna en el municipio de Fusagasugá sin distinción de condición socioeconómica ni grado de escolaridad. El factor que predominó fue la baja producción láctea que puede deberse más a las pobres condiciones alimenticias durante el embarazo que a una patología específica, lo que obligó a implementar medidas o campañas de alimentación balanceada y buenos hábitos en las madres del municipio. Se determinó que $22,7 \%$ de los niños necesitaron el servicio del banco de leche materna, logrando que lo pudieran obtener de manera adecuada y en la misma zona de nacimiento, sin necesidad de desplazarse con las subsiguientes consecuencias que ello desencadena en la familia y en el mismo recién nacido.

\section{REFEREN CIAS}

1. Organización Mundial de la Salud. Estrategia mundial para la alimentación del lactante y del niño pequeño. Duración óptima de la lactancia materna exclusiva. Organización Mundial de la Salud; 2001. p. 5.

2. Sáenz-Lozada M, Camacho-Lindo Á. Prácticas de Lactancia Materna y Alimentación Complementaria en un Jardín Infantil de Bogotá. Rev Salud Pública. 2007;9(4):587-94.

3. Vázquez Román S, Alonso Díaz C, Medina López C, Bustos Lozano G, Martínez Hidalgo MV, Pallás Alonso CR. Puesta en marcha del banco de leche materna donada en una unidad neonatal. An Pediatr. 2009;71(4):343-8.

4. Machado RdS, Campos Calderón CP, Montoya Juárez R, Schmidt RioValle J. Experiencias de donación de leche humana en Andalucía-España: un estudio cualitativo. Enferm Glob. 2015;14(37):114-24.

5. Pittas TM, Dri CF. El diálogo entre la salud y la política exterior en la cooperación brasileña en bancos de leche humana. Ciênc Saúde Coletiva. 2017;22(7):2277-86

6. Ramiro González MD, Ortiz Marrón H, Arana Cañedo-Argüelles C, Esparza Olcina MJ, Cortés Rico O, Terol Claramonte M, et al. Prevalencia de la lactancia materna y factores asociados con el inicio y la duración de la lactancia materna exclusiva en la Comunidad de Madrid entre los participantes en el estudio ELOIN. Anales de Pediatría (English Edition). 2018;89(1):32-43.

7. Baquero H, Velandía L. Nutrición del prematuro. Rev Precop CCAP Colomb. 2010;9(4):22-32.

8. Sara Macías D, Rodríguez S, Ronayne de Ferrer P, Sara Macías D. Leche materna: Composición y factores condicionantes de la lactancia. Arch Argent Pediatr. 2006;104(5):423-30.

9. Organización Panamericana de la Salud. Alimentacion y nutricion del niño pequeño. Memoria de la reunion subregional de los paises de sudamerica Managua, Nicaragua Organización Panamericana de la Salud; 2010. p. 4.
10. Mupsilonller MJ, Paul T, Seeliger S. Necrotizing enterocolitis in premature infants and newborns. Journal of neonatal-perinatal medicine. 2016;9(3):233-42.

11. Vohr BR, Poindexter BB, Dusick AM, McKinley LT, Higgins $\mathrm{RD}$, Langer JC, et al. Beneficios persistentes de la leche materna ingerida por infantes con peso extremadamente bajo al nacer en la unidad de cuidados intensivos neonatales. p. 3.

12. Organización Mundial de la Salud. Razones médicas aceptables para el uso de sucedáneos de leche materna Organización Mundial de la Salud; 2009. p. 6.

13. Ministerio de Salud y Protección Social. Banco de leche humana BHL en Colombia. Colombia: Ministerio de Salud y Protección Social; 2015. p. 8.

14. Fonseca Acevedo I, Torrez Bracamonte NR, Pineda Happer S. Valor calórico de la leche materna almacenada en el banco de leche y su relación con hábitos alimentarios de las madres donantes del Hospital Bertha Calderón, periodo Noviembre - Diciembre 2015 [Tesis]. Managua, Nicaragua: Universidad Nacional Autónoma de Nicaragua; 2016.

15. Bejarano Roncancio J. El banco de leche humana y el lactario hospitalario. Rev Gastrohnup. 2013;15(1 Suplemento 2):S30-40.

16. Espín Brito DC. Implementar un banco de leche en el servicio de neonatología del Hospital Less Ambato durante el periodo abril 2016 a septiembre 2016 [Tesis]. Ecuador: Universidad Regional Autónoma De Los Andes; 2016.

17. Cánepa M. Un modelo de comparación horizontal: La Red Iberoamericana de bancos de leche humana (BHL). Estudio de caso. Madrid, España: Organización Panamericana de la Salud, Organización Mundial de la Salud; 2011.

18. Secretaria General Iberoamericana. Programa IberBLH: objetivos [Internet]. Secretaria General Iberoamericana; 2008 [cited 2017 noviembre 21]; Available from: https:// www.iberblh.icict.fiocruz.br/index.php?option $=\mathrm{com}_{-}$ content $\&$ view $=$ article\&id $=47 \&$ Itemid $=27$.

19. Ministerio de Sanidad SSeI, Eusko Jaurlaritzaren Argitalpen Zerbitzu Nagusia, Servicio Central de Publicaciones del Gobierno Vasco. Guia practica lactancia materna en el lugar de trabajo. España: Administración de la Comunidad Autónoma del País Vasco, Departamento de Salud; 2017. p. 316.

20. Subdirección de Salud Nutricional AyB, Ministerio de Salud y Protección Social. Articulacion estrategias bancos de leche humana - Institucion Amiga de la mujer y la infancia. Colombia: Ministerio de Salud y Protección Social; 2015. p. 19.

21. PRINCIPIOS DE BRASILIA: Principios rectores para el desarrollo de la atención en salud mental en las Américas. Brasilia2005. p. 5.

22. Ministerio de Salud y Protección Social. Impacto positivo en implementación de Bancos de Leche Humana en el país [Internet]. Colombia: Ministerio de Salud y Protección Social; 2013 [citado 2017 noviembre 20]; Disponible en: https://www.minsalud.gov. co/Paginas/Bancos-de-Leche-Humana.aspx.

23. Departamento Nacional de Planeación. Bases del plan nacional de desarrollo 2014-2018 Colombia: Departamento Nacional de Planeación; 2013. p. 793. 